\title{
Un bilan de l'évolution de l'intérêt des médecins québécois pour les maladies infectieuses dans les périodiques médicaux (1826-1899)
}

\section{André Paradis}

Volume 43, numéro 1, été 1989

URI : https://id.erudit.org/iderudit/304766ar

DOI : https://doi.org/10.7202/304766ar

Aller au sommaire du numéro

Éditeur(s)

Institut d'histoire de l'Amérique française

ISSN

0035-2357 (imprimé)

1492-1383 (numérique)

Découvrir la revue

Citer cet article

Paradis, A. (1989). Un bilan de l'évolution de l’intérêt des médecins québécois pour les maladies infectieuses dans les périodiques médicaux (1826-1899). Revue d'histoire de l'Amérique française, 43(1), 63-91.

https://doi.org/10.7202/304766ar
Résumé de l'article

S'appuyant sur une recension des textes parus sur les maladies infectieuses dans les périodiques médicaux québécois du $\mathrm{XIX}^{\mathrm{e}}$ siècle, cet article conclut à une évolution en quatre phases de l'intérêt des médecins pour ces maladies entre 1826 et 1899. Il cherche à en rendre compte par des facteurs afférents tant à l'histoire sociale qu'institutionnelle et scientifique. Il illustre aussi l'évolution du degré d'implication des médecins québécois dans leurs propres organes de presse, souligne l'importance croissante des emprunts faits à la littérature étrangère et montre des différences significatives dans le volume de production et la distribution des textes francophones et anglophones. Il y est démontré que les différentes pathologies infectieuses n'ont pas toutes la même visibilité et que l'intérêt qu'elles suscitent varie en fonction de caractéristiques intrinsèques et circonstancielles propres à chaque maladie. Globalement, cet article insiste sur le fait que les infections jouent un rôle structurant dans l'institutionnalisation de la profession médicale au XIX ${ }^{\mathrm{e}}$ siècle et que c'est largement autour d'elles que s'articule aussi le discours scientifique des médecins. 


\title{
UN BILAN DE L'ÉVOLUTION DE L'INTÉRÊT DES MÉDECINS QUÉBÉCOIS POUR LES MALADIES INFECTIEUSES DANS LES PÉRIODIQUES MÉDICAUX (1826-1899)
}

\author{
ANDRÉ PARADIS \\ Département de philosophie \\ Centre de recherche en études québécoises \\ Université du Québec à Trois-Rivières
}

\begin{abstract}
RÉSUMÉ
S'appuyant sur une recension des textes parus sur les maladies infectieuses dans les périodiques médicaux québécois du XIXe siècle, cet article conclut à une évolution en quatre phases de l'intérêt des médecins pour ces maladies entre 1826 et 1899 . Il cherche à en rendre compte par des facteurs afférents tant à l'histoire sociale qu'institutionnelle et scientifique. Il illustre aussi l'évolution du degré d'implication des médecins québécois dans leurs propres organes de presse, souligne l'importance croissante des emprunts faits à la littérature étrangère et montre des différences significatives dans le volume de production et la distribution des textes francophones et anglophones. Il y est démontré que les différentes pathologies infectieuses n'ont pas toutes la même visibilité et que l'intérêt qu'elles suscitent varie en fonction de caractéristiques intrinsèques et circonstancielles propres à chaque maladie. Globalement, cet article insiste sur le fait que les infections jouent un rôle structurant dans l'institutionnalisation de la profession médicale au XIXe siècle et que c'est largement autour d'elles que s'articule aussi le discours scientifique des médecins.
\end{abstract}

\begin{abstract}
Based on a recension of texts that appeared regarding infectious diseases in Quebec's medical periodicals of the nineteenth century, this article concludes that the physician's interest in these diseases evolved in four phases between 1826 and 1899. It seeks to explain this through factors pertaining as much to social as to institutional and scientific history. It also illustrates the increasing involvement of Quebec's physicians in their own scientific reviews, emphasizes the growing importance of ideas borrowed from foreign publications and shows significant differences in the volume of production and the distribution of French and English texts. The article also demonstrates that the various infectious pathologies do not share the same visibility and that the interest they arouse varies according to the intrinsic and circumstantial attributes specific to each disease. Globally speaking, this article lays stress on the fact that infections played a shaping role in the institutionalization of the medical profession in the nineteenth century and that it was mainly around these infections that the physicians elaborated their scientific discourse.
\end{abstract}

\section{INTRODUCTION}

La vocation première de la médecine coloniale ayant été de suivre les armées, on ne doit pas s'étonner que la chirurgie ait été son premier

RHAF, vol. 43, no 1, été 1989 
fleuron: réduire les fractures, recoudre, cautériser et panser les plaies ouvertes, ligaturer les artères, juguler les hémorragies et amputer les membres tuméfiés et gangrenés, telles devaient être les compétences des médecins militaires des XVIIe et XVIIIe siècles. Avec l'intensification de la colonisation et du commerce maritime apparait toutefois, au milieu du XVIIIe siècle, une autre préoccupation majeure: celle de contrôler et de mieux connaître les maladies «pestilentielles» qui déciment, outre les garnisons, les agglomérations de colons et les populations autochtones. L'importance de cette préoccupation se trouve attestée dès 1721 par une ordonnance de quarantaine de l'intendant Bégon, laquelle sera reconduite en 1759 , puis, en 1785 , neuf ans après le mandat confié à Badelard, par une enquête médicale sur le mal de la Baie Saint-Paul (syphilis) ordonnée par le général Hamilton. En 1795, l'année même de la découverte de l'immunisation vaccinale par Jenner, Carleton impose, à son tour, par le biais de l'Assemblée, une quarantaine, mais cette fois après avoir consulté un comité ad hoc de chirurgiens, membres des bureaux d'examinateurs nouvellement mis en place sous son instigation. Dès lors s'amorce, au seuil du XIXe siècle, une collaboration plus ou moins concertée entre la profession médicale naissante et le gouvernement colonial qui visera à assurer l'inspection médicale occasionnelle des navires, la diffusion de la vaccination antivariolique dans la population civile et la mise sur pied de dispensaires et d'hôpitaux consacrés surtout à la garde et au soulagement des immigrants contagieux et sans ressources.

L'importance que prendront par la suite les maladies infectieuses aux yeux de l'administration politique de la colonie sera proportionnelle à l'ampleur même de l'immigration, à l'expansion des villes, aux carences de plus en plus évidentes du réseau hospitalier et finalement aux avatars du capitalisme commercial et industriel qui mettra bien du temps à financer la gestion politique et le contrôle médical de ses propres débordements. L'absence de bureaux d'hygiène municipaux permanents avant 1875 , la précarité des infrastructures urbaines, la paupérisation et la malnutrition, le chômage et la prostitution, la densification des quartiers ouvriers et les conditions de logement et de travail insalubres compteront alors parmi les principaux facteurs favorisant la propagation des infections. De la création du lazaret de la Grosse-Ile destiné à intercepter les cholériques de 1832 jusqu'à la normalisation des mesures d'hygiène publique à la toute fin du XIXe siècle, les instances politiques seront donc amenées par la force des choses à solliciter de plus en plus l'aide des médecins pour préserver et promouvoir ce qu'on finira par appeler la «santé publique». Durant toute cette période, les maladies infectieuses contribueront non seulement à structurer au premier chef l'institution médicale ${ }^{1}$, mais c'est aussi largement autour

1 Je fais allusion en particulier ici à l'impact des épidémies dans la création de commissions d'enquête médicales, de bureaux d'hygiène provinciaux et municipaux, de quarantaines, d'hôpi- 
d'elles que viendra s'articuler le discours scientifique des médecins. De 1827 à 1899 , pas moins de 3500 textes paraîtront en effet sur le seul thème des maladies infectieuses dans les périodiques médicaux spécialisés du Québec, ce qui équivaut grosso modo au quart de l'information médicale qui s'y trouve diffusée. C'est dire que parallèlement à la chirurgie, à l'obstétrique et à la gynécologie, ce sont surtout les infections qui pousseront les médecins du XIXe siècle à symboliser leurs pratiques et à les légitimer. Les infections seront à la fois le fer de lance et le talon d'Achille de la profession médicale. Tout en stimulant de façon étonnante le développement de la pathologie clinique et l'imagination thérapeutique ${ }^{2}$, elles ne cesseront pour autant de déjouer la kyrielle des innombrables traitements à prétention curative et de rappeler aux médecins les limites et la fragilité de leur art.

L'intention de cet article est précisément de suggérer, sur la base de considérations quantitatives et à partir du dépouillement des périodiques médicaux, un aperçu de l'évolution de l'intérêt des médecins québécois pour les maladies infectieuses de 1827 à 1899. Sans doute le périodique médical n'est-il pas l'unique mesure de cet intérêt. Mais il en est un témoin d'autant plus fiable et intéressant qu'il s'alimente des écrits d'une bonne fraction de l'élite médicale locale, qu'il reflète assez bien ses préoccupations courantes en ce domaine, que c'est lui qui permet dans une bonne mesure l'acculturation des idées médicales étrangères et que c'est aussi le seul type de publication qui nous autorise, dans la longue durée, à des comparaisons significatives. Les autres types de publications médicales du XIXe siècle, tels les traités de médecine, les monographies spécialisées, les rapports d'hôpitaux et les dossiers cliniques sont trop rares ou trop disparates en effet pour nous permettre d'observer des fluctuations tant soit peu significatives dans les centres d'intérêt des médecins. Par ailleurs, le périodique médical, qui tient largement du prestige, de la distinction personnelle et de la polémique à ses débuts, ce qui explique en partie sa précarité, devient vite à partir des années 1850 un véritable organe de mobilisation professionnelle et, dans le dernier tiers du siècle, un moyen prépondérant d'intégrer l'enseignement médical, la clinique hospitalière et la vocation savante des associations médicales. Il s'agit donc d'un matériau

taux, de bureaux de vaccination, etc. Consultez en particulier à ce propos Claudine Pierre-Deschênes, «Santé publique et organisation de la profession médicale au Québec 1870-1918», Revue d'histoire de l'Amérique française, 35,3 (décembre 1981): 355-375; M. Farley, O. Keel et C. Limoges, «Les commencements de l'administration montréalaise de la santé publique (18651885)», Revue d' histoire des sciences, des techniques et de la médecine au Canada, 6,1 (janvier 1982): 24-46, 6,2 (mai 1982): 85-109.

Si on s'en tient aux périodiques médicaux, on peut estimer en effet à plusieurs centaines les types de traitements mécaniques (tubage, bains, compression, etc.), chirurgicaux et pharmacologiques pratiqués et/ou connus des médecins québécois au XIXe siècle. Non encore expérimentale, la médecine n'en est pas moins, à première vue, étonnamment «exploratoire». Reste évidemment à vérifier dans quelle mesure les archives hospitalières confirment ce foisonnement de thérapies dont la plupart sont empruntées à la médecine européenne. 
clé pour qui veut comprendre tant l'évolution des centres d'intérêt des médecins que l'évolution des conceptions médicales elles-mêmes.

Dans le cadre de cet article, le concept d'intérêt référera essentiellement à l'intérêt qu'ont eu les médecins du XIXe siècle de produire et/ ou de mettre en circulation des textes sur les infections qu'on peut $a$ posteriori dénombrer et supputer statistiquement. Il s'agit donc avant tout d'un concept opératoire, qui a ses propres limites, et qui, comme tel, ne renvoie à aucune motivation psychologique pas plus qu'il ne connote que certains textes sont plus «intéressants» que d'autres. Chaque texte, qu'il soit signé ou anonyme et quel qu'en soit le volume ou le genre, correspond ici à une unité quantitative d'information. On y verra peut-être un inconvénient mais il est important de retenir que les inégalités de volume et de genre se répartissent de façon relativement égale sur l'ensemble du siècle, ce qui nous autorise à des conclusions valables. Nous convenons cependant qu'une analyse plus fine, qui tiendrait rigoureusement compte de la différence des genres et des volumes, permettrait de tirer des conclusions encore plus nuancées. Les genres auxquels nous faisons allusion sont les articles de fond, les rapports de comités, les lettres, les comptes rendus et commentaires de volumes et d'articles, les études de cas cliniques, les procès-verbaux de séances de sociétés et les entrefilets pour autant qu'ils portent sur la pathologie, la thérapeutique, l'épidémiologie, l'encadrement médical ou la prophylaxie sociale des maladies infectieuses. Par maladies infectieuses, nous entendons par ailleurs les maladies contagieuses connues des médecins du XIXe siècle, y compris certaines maladies transmissibles sexuellement, mais aussi des maladies d'origine virale, bactérienne ou parasitaire plus rares ou en voie de régression, telles la peste, la lèpre, l'érysipèle, la rage, la gale et le tétanos. Des types d'infections dont le profil clinique est mal défini ou qui n'apparaissent d'aucune façon dans la nosographie du XIXe siècle, comme la poliomyélite ou le chlamydia, nous n'avons évidemment pas tenu compte.

En tout et partout, nous avons retenu 27 types d'infection et répertorié 3393 textes dans 19 périodiques médicaux spécialisés ${ }^{3}$. Ces textes

\footnotetext{
3 Les périodiques que nous avons dépouillés sont mentionnés à même les données bibliographiques dans André Paradis et Hélène Naubert, avec la collaboration de Denis Goulet, Recension bibliographique: les maladies infectieuses dans les périodiques médicaux québécois du XIXe siècle (1), Trois-Rivières, Centre de recherches en études québécoises, Université du Québec à Trois-Rivières, coll. «Matériaux pour l'histoire de la médecine québécoise», 1988. Nous n'avons pas inclus toutefois dans cette publication les maladies transmises sexuellement dont nous tiendrons compte ici. Par ailleurs, nous avons omis dans notre recension la fièvre puerpérale et les septicémies dont il est assez souvent question dans les périodiques du XIXe siècle. Pour des raisons de temps et de moyens matériels, certains numéros de périodiques médicaux, plus rares, ont échappé à notre recension, notamment le Canada Lancet (1863-1864) et les volumes 1867-1868 et 1868-1869 du Canada Medical Journal and Monthly Record of Medical and Physical Science. Nous prions donc le lecteur de pondérer ces manques qui se traduisent dans certains de nos graphiques par une chute de la courbe pour ces trois années.
} 
se trouvent surtout concentrés dans le dernier tiers du XIXe siècle. Mille deux cent cinq d'entre eux sont signés (soit 36\%) dont 617 (soit 18\%) par des médecins pratiquant au Québec. Des 191 signataires québécois ${ }^{4}, 115$ (soit $60 \%$ ) sont anglophones et 76 (soit $40 \%$ ) sont francophones. Quarante signataires seulement ont entre trois et neuf textes à leur crédit et 15 en ont 10 et plus. Des 55 médecins qui ont plus de trois textes, 32 sont anglophones et 23 sont francophones. Sans extrapoler indûment, ces chiffres nous donnent déjà une bonne idée de l'ampleur des effectifs et de la ventilation des médecins rédacteurs du XIXe siècle par rapport à leur langue d'expression. Il va sans dire que la plupart des textes signés sont des articles plutôt théoriques mais il peut s'agir aussi de rapports sur des cas de clinique médicale ou chirurgicale. La majorité des textes recensés, soit approximativement $45 \%$ de notre documentation, se présentent toutefois sous forme de commentaires ou de comptes rendus anonymes de volumes et d'articles locaux ou étrangers qui vont du résumé succinct ou anecdotique à la reproduction quasi littérale en passant par tous les degrés de la glose libre. Après 1880, il arrive de temps à autre que les comptes rendus, plus élaborés, soient signés ou initialisés sans ambiguité possible sur l'identité de l'auteur. Le texte typique du genre compte rendu varie entre une et trois pages. Il en va généralement de même des articles. Certains articles cependant peuvent exceptionnellement avoir entre 4 et 12 pages quand ils ne sont pas livrés par tranches.

Les textes que nous avons recensés réfèrent majoritairement, dans à peu près huit cas sur dix, à la thérapeutique et à la pathologie des infections, le reste se distribuant sur la prophylaxie médicale, l'épidémiologie des maladies courantes, l'encadrement hospitalier et les mesures de prévention et d'hygiène publique. Cette distribution se comprend assez bien quand on sait que les périodiques médicaux sont généralement entre les mains de cliniciens dont les préoccupations sont avant tout pratiques: elles visent l'établissement d'un diagnostic aussi précis que possible et le soulagement des malades. En regard des différentes pathologies concernées, ces textes se répartissent comme suit: tuberculose: 744; fièvre typhoïde: 348 ; diphtérie: 348 ; variole: 322 ; syphilis: 285 ; choléra: 168 ; rougeole: 144 ; pneumonie: 117 ; fièvre scarlatine: 102; gonorrhée: 98 ; coqueluche: 76; blennorragie: 59; érysipèle: 54; dysenterie: 48; rougeole 44; pleurésie: 39; grippe: 36; bronchite: 31 ; tétanos: 28; fièvre jaune: 23 ; rage: 22 ; lèpre: 18 ; chancre mou: 16 ; typhus: 13; malaria: 13; peste: 5; gale: 4 . A ces textes, il faut en rajouter 188 qui portent simultanément sur plusieurs types d'infections, sur les «fièvres» en général ou sur la microbiologie dans son rapport à l'antisepsie et au diagnostic des infections.

4 Il y a quelques cas litigieux où nous n'avons pas pu conclure avec certitude que les signataires pratiquaient bien au Québec. 
L'analyse de notre corpus nous a permis de dégager certaines caractéristiques importantes et significatives dans la production d'informations sur les maladies infectieuses, en particulier par rapport 1) au rythme de la production globale d'information sur l'ensemble du siècle 2) à l'évolution du nombre de textes signés par les médecins québécois par rapport au nombre total de textes parus 3) à l'évolution comparative de la production des médecins francophones et anglophones 4) et à l'évolution de la production d'information par rapport à chacune des grandes pathologies infectieuses. Ces caractéristiques, traitées dans l'ordre, formeront le corps de notre texte.

\section{LE BILAN}

La production globale d'information sur l'ensemble du siècle

Le graphique 1 indique, pour chaque année, le nombre total de textes parus sur les maladies infectieuses, quels qu'en soient le genre littéraire, le format ou la provenance (locale ou étrangère), qu'ils soient signés ou anonymes. Ce graphique illustre assez nettement que la production totale d'information sur les maladies infectieuses se distribue sur quatre paliers qui constituent autant de phases de production bien démarquées: une première, éphémère, qui couvre les années 1826-1827; une seconde qui, après un temps d'éclipse attribuable à la disparition du périodique, va de 1844 à 1871; une troisième, particulièrement prolifique, qui s'étend de 1872 à 1894; et une quatrième qui débute en 1895 et qui correspond grosso modo à l'entrée de la médecine québécoise dans l'ère de la médecine techno-scientifique. Ces quatre paliers de productions ne sont assurément pas aléatoires. Nous tenterons de

GRAPHIQUE 1

Évolution du nombre total de textes (1826-1899)

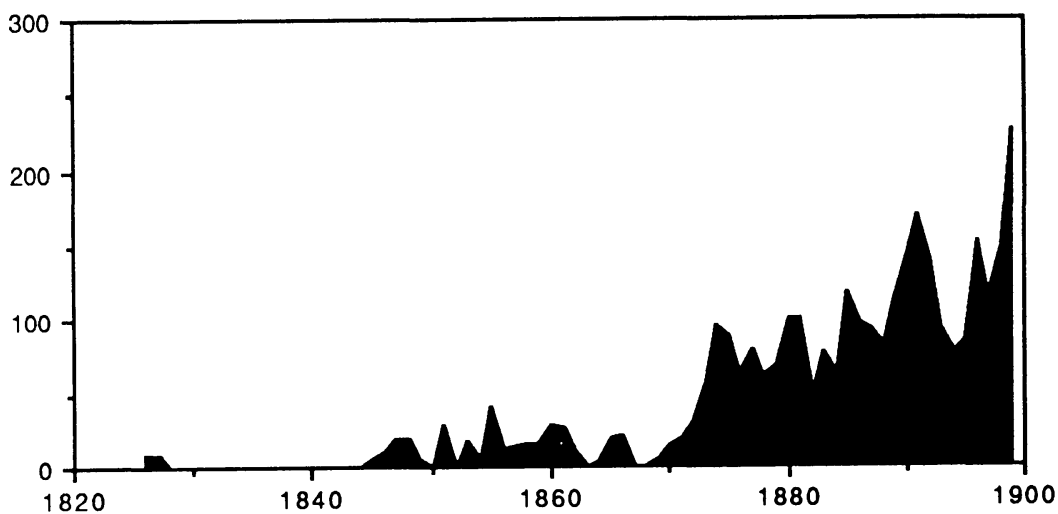


montrer d'abord brièvement et à grands traits en quoi ils sont solidaires de développements dans la société globale et dans les infrastructures de la profession médicale.

\section{a) La phase 1826-1844}

Très modeste, la production de 1826 et 1827 totalise 8 textes/ année. Il s'agit essentiellement de rapports saisonniers qui font état de la santé publique, des épidémies les plus courantes de rougeole, de grippe et de variole et des tentatives faites pour promouvoir la vaccination antivariolique. Il ne s'agit donc pas d'articles spécialisés sur la pathologie des infections comme on en aura dès la phase suivante. Cette première vague de pièces documentaires se situe dans le prolongement d'un nombre appréciable d'initiatives individuelles ou conjointes entre les médecins et l'État pour contrer les épidémies ${ }^{5}$. Elle n'est pas sans rapport avec la croissance subtantielle de l'immigration qui prévaut depuis 1817 et avec le fait que les médecins des milieux urbains, déjà sollicités par le pouvoir politique, tendent à se regrouper et à développer, au niveau même de leur pratique professionnelle, un certain leadership social. Même si leurs entreprises sont encore fragiles, les médecins manifestent de plus en plus clairement leur intention d'accéder, comme groupe, à une plus grande visibilité sociale et à une conception plus critique de la médecine comme science. La Société médicale de Québec naît en effet en 1826 sous le signe d'une collaboration de principe entre les médecins des deux principaux districts du Bas-Canada. Le Journal de Médecine de Québec, qui est l'organe officiel de la Société, voit le jour la même année et traduit assez bien dans ses politiques liminaires l'optimisme intellectuel et l'espèce d'euphorie «empiriste» qui semble alors gagner la profession ${ }^{6}$. Formée à l'étranger et gagnée à la mentalité universaliste, la nouvelle élite médicale, avec Tessier et $\mathrm{F}$. Blanchet à sa tête, envisage le recours à la littérature médi-

\footnotetext{
5 Mentionnons, entre autres, les efforts du docteur Longmore dans le district de Québec (1802) et du docteur von Iffland dans le district de Gaspé (1821) pour répandre la vaccination antivariolique dans la population civile et la mise sur pied de bureaux de vaccination à Québec $(1817,1821)$. En 1829, la Société médicale de Québec s'affiliera à la London Vaccine Institution (C.- M. Boisssonneault, Histoire de la faculté de médecine de Laval (Québec, Presses de l'Université Laval, 1953), 124. Il y a aussi la passation d'une série de lois (1802, 1809, 1812, 1817 , 1823) visant à améliorer et à rendre opérationnelles les mesures de quarantaine adoptées en 1795 et la création de dispensaires et d'hôpitaux destinés en partie ou en totalité au traitement des contagieux: nommément l'hôpital de l'Île-aux-Ruaux, située au nord-est de l'île d'Orléans (1817), l'Hôpital des fébricitants de Pointe-Lévis (1830), l'Hôpital des Émigrés de Québec (1823) et la House of Recovery de Montréal, premier noyau du Montreal General Hospital, qui sera mis sur pied pour venir en aide aux immigrants atteints du typhus lors de l'épidémie de 1817. C'est dans le même esprit d'urgence et de collaboration entre les médecins et les instances politiques que seront d'ailleurs formés les premiers bureaux de santé temporaires à Québec $(1823,1832)$ et à Montréal $(1839,1840)$. Ces références factuelles, ainsi que d'autres dans notre texte, sont tirées d'une chronologie critique actuellement en voie de préparation et menée en collaboration avec Denis Goulet de l'Université de Montréal.

6 Voir l'introduction du journal rédigée par F.-X. Tessier.
} 
cale britannique, américaine et française comme un levier pour donner plus de légitimité et de crédibilité à la profession. La démocratisation des bureaux d'examinateurs, qui prendra effet avec la loi de 1831, viendra bientôt couronner cette époque prospère de la médecine québécoise. L'État reconnaîtra désormais aux médecins le droit de nommer eux-mêmes leurs propres représentants ${ }^{7}$.

L'absence de périodiques et donc de textes sur les infections entre 1828 et 1844 est vraisemblablement attribuable à la combinaison de plusieurs facteurs, dont en particulier les rivalités entre médecins francophones et anglophones ${ }^{8}$, la crise économique des années 1830 , la rébellion de 1837, la densité encore relativement faible des villes et la précarité des infrastructures médicales hospitalières et professionnelles. Mais le facteur le plus déterminant de la désaffection des médecins pour la vie intellectuelle durant cette période ${ }^{9}$ semble bien être la marée montante de l'immigration ${ }^{10}$ qui déferle sur la colonie et qui, à sept reprises entre 1832 et 1854 , amènera avec elle son tribut de cholériques et de typhiques. L'historiographie québécoise nous permet d'évaluer à environ $32000^{11}$ le nombre de victimes décédées du choléra ou du typhus durant ces sept épidémies pour les seuls districts de Québec et de Montréal. Encore ne s'agit-il là que d'une proportion restreinte des malades et des contagieux hospitalisés ${ }^{12}$. Nul doute que le corps médical, lui-même ébranlé par la contagion, dut être grandement mobilisé par les soins à donner aux malades et préoccupé par le manque de ressources disponibles dans la colonie. Le lazaret de la Grosse-Isle est un exemple frappant, dès le début des années 1830, du retard accusé par l'institution médicale sur le développement démographique. Dire que les conditions d'accueil et de vie qui y règnent sont déplorables

7 Voir à ce propos J. Bernier, La médecine au Québec, naissance et évolution d' une profession (Québec, Les Presses de l'Université Laval, 1989), et Barbara Tunis, «Medical Licensing in Lower Canada», Medecine in Canada Society (Montréal, McGill-Queen's University Press, coll. «Historical Perspectives», 1981), 137-163.

8 Cette rivalité s'amorce vraisemblablement avec la monopolisation des postes par les francophones sur les bureaux d'examinateurs. Elle ira grandissante avec les troubles de 1837-1838, la revendication d'un Collège indépendant et souverain par les médecins francophones sous le leadership de W. Nelson, revenu d'exil, et avec l'obstruction de McGill aux revendications des professeurs de l'École de médecine et de chirurgie dans les années 1850 . On en retrouve des échos lors de la fondation de l'Association médicale canadienne et sur la fin du siècle avec l'anticipation du fameux bill «centralisateur» de Roddick.

9 La Société médicale de Québec, fondée en 1826, semble disparaitre à toute fin utile vers 1832, peu de temps après le Journal de médecine de Québec; les bureaux de vaccination et les dispensaires ne font pas long feu eux aussi.

${ }_{10}$ L'immigration massive des Irlandais (on en compte entre 5000 et 30000 chaque année entre 1831 et 1838 et 90000 pour la seule année 1847) mettra en effet à rude épreuve la capacité d'accueil des sociétés de bienfaisance et des hôpitaux traditionnels.

11 Soit deux fois la population de Québec à l'époque

12 Les épidémies importantes parallèles au choléra et au typhus sont l'influenza (1826, $1830,1836,1844)$, la variole $(1820,1824,1830,1833,1842)$ et la fièvre typhoïde $(1826)$. 
relève de l'euphémisme ${ }^{13}$. L'hôpital des Émigrés en est aussi un exemple patent. Le docteur J. Douglas rappelle lapidairement à propos de cet hôpital complètement désorganisé par la première épidémie de choléra en 1832: «It was a scene of drunkenness, licentious and open robbery.» ${ }^{14}$ L'hôpital de la Marine se retrouvera lui aussi complètement débordé lors de l'épidémie de typhus de $1847^{15}$, tout comme d'ailleurs le Montreal General Hospital. Tant sur les plaines d'Abraham qu'autour de l' «Hôpital anglais» on sera contraint d'édifier plusieurs centaines de tentes pour abriter les malades. Ce n'est certainement pas sans raison que les historiens $\mathrm{H}$. Agnew et Geoffrey Bilson ${ }^{16}$ attirent l'attention sur la réputation douteuse qui frappe l'institution médicale à compter des années 1830 . Si on en croit les témoignages du chirurgien Shepherd et de l'anesthésiologiste Howell ${ }^{17}$, on peut penser que la réputation de «mouroirs» des hôpitaux, encore existante sur la fin du siècle, s'enracine dans cette période assez trouble et difficile de l'histoire médicale ${ }^{18}$. Si les épidémies n'en sont pas l'unique cause, on peut penser qu'elles en sont l'un des principaux révélateurs. En somme, si la médecine française, en dépit de conditions également difficiles, fait alors des progrès sensibles dans la clinique des maladies infectieuses avec Louis, Bretonneau, Lagneau et Ricord, on peut dire que la période 1831-1844 en est une au contraire peu propice au développement des connaissances médicales au Bas-Canada.

\section{b) La phase 1844-1871}

La phase 1844-1871 se caractérise, du point de vue du volume de la production intellectuelle sur les infections, par des fléchissements et des remontées répétés, mais d'un même ordre de grandeur, qui font qu'au seuil des années 1870 cette même production n'est guère supérieure à ce qu'elle était au cours des vingt années précédentes. Il s'agit donc d'une phase de production précaire mais néanmoins soutenue et qui montre une hausse appréciable en regard des années 1826-1827. Le volume des textes produits annuellement fait plus que doubler et oscille

\footnotetext{
13 Un vidéo sur la Grosse-Isle produit avec la participation du docteur Leblond et présenté à Montmagny au cours de l'été 1988 donne une bonne idée de l'insalubrité des lieux lors des épidémies de typhus et de choléra. Voir aussi à ce propos Marianna O'Gallagher, Gosse-Ile, porte d'entrée du Canada, 1832-1937 (Québec, Carraig Books, 1987).

${ }_{14}$ Voir W. B. Howell, Medicine in Canada (Clio Medica, Paul B. Hoeber, 1933), 68.

15 C.-M. Boissonnault, op. cit., 100.

16 G. Bilson, «Canadian Doctors and the Cholera», S. E. D. Shortt, Medicine in Canadian Society - Historical Perspectives (Montréal, McGill-Queen's University Press, 1981), 119: «Some of the major efforts toward achieving professionnal standing were made between the early 1830s and the later 1860 s, but they were made at a time when the doctors were losing public esteem.»

17 Voir W. B. Howell, F. J. Shepherd - Surgeon, his Life and Times (Toronto and Vancouver, J. M. Dent and Sons Ltd, 1934), 23-25; F. J. Shepherd, Origin and History of the Montreal General Hospital (The Gazette Printing, 1919), 13.

18 Dès 1849 , une commission présidée par W. Nelson sera d'ailleurs formée pour faire enquête sur l'état des asiles et des hôpitaux.
} 
autour d'une moyenne de 20 . La très grande sinuosité de la courbe s'explique sans doute du fait que l'intérêt porté aux maladies infectieuses semble varier selon le moment et l'intensité des épidémies. Les sommets de production se situent en effet autour des années 1847, 1849, $1851,1852,1854$ et 1863 , années où sévissent le typhus, le choléra, la diphtérie et la variole. Même si le sommet de 1860 fait exception, cette présomption se trouve raisonnablement confirmée durant la phase suivante, puisque les sommets de 1872-1876, de 1885 et de 1889-1893 correspondent eux aussi à l'occurrence d'épidémies de variole, de diphtérie et de fièvre scarlatine particulièrement sévères.

On notera que durant cette phase, surtout entre 1845 et 1860 , les périodiques médicaux réussissent à peine à se maintenir eux aussi. Ceux qui durent plus de cinq ans se résument en effet au Medical Chronicle (1852-1859) et au Canada Medical Journal and Monthly Record of Medical and Surgical Science (1864-1872) ${ }^{19}$. La très grande majorité des périodiques de cette phase n'excèdent pas en fait deux ou trois années d'existence. Certains ne titrent même pas pendant une année. Néanmoins, il en est toujours au moins un pour prendre la relève. On pourrait évidemment s'interroger sur l'absence de progression significative dans la production d'information sur les infections entre 1845 et 1872 , alors que sont mises en place les premières écoles de médecine francophones et que naît le Collège des médecins et chirurgiens de la province de Québec ${ }^{20}$. Il faudrait alors montrer que le développement de la médecine se fait alors presque exclusivement dans le secteur anglophone, ce qu'on pourrait illustrer du point de vue du nombre des inscriptions et des diplômés en médecine, de la fondation des nouveaux

19 Les autres périodiques de la période 1844-1871 sont, dans l'ordre, le Montreal Medical Gazette (1844-1845), le British American Journal of Medical and Physical Science (1845-1850), la Lancette Canadienne (1847), le British American Medical and Physical Journal (1850-1852), le Montreal Medical Gazette (1852-1853), qui connaît une sorte de résurrection éphémère, le British American Journal (1860-1862) et le Canada Lancet (1863-64).

20 Ầ mon sens, l'incorporation du Collège des médecins et chirurgiens, revendiquée surtout par les médecins francophones pour contrebalancer l'influence du Collège McGill, fut en bonne partie concédée parce qu'il était devenu évident que le gouvernement colonial ne réussirait pas à gérer «démocratiquement» la crise sociale (particulièrement aiguisée par les famines, les épidémies, les troubles politiques, la forte croissance démographique, la prolétarisation et l'augmentation des «marginalités dépendantes» (orphelins, fous, mères célibataires, mendiants, malades, etc...) sans s'assurer le concours politique des médecins et des communautés religieuses francophones. L'arrivée d'une nouvelle vague d'immigrants irlandais, anticipée depuis la famine de la pomme de terre de 1845, certaines divisions internes à la fraction anglophone de la profession depuis 1843 (Badgley, McNider, etc...) et la grande épidémie de typhus de 1847 ont pu exercer aussi une certaine influence sur la finalisation du consensus entre médecins et sur la conclusion d'une nouvelle alliance entre l'État et le corps médical unifié. En d'autres mots, l'incorporation et l'unification libérale de la profession, étonnamment précoces par rapport à celles du HautCanada (1869), des États-Unis et même de la métropole anglaise, sont peut-être beaucoup moins dues à la vitalité interne de la profession qu'à la pression des circonstances et des transformations structurelles que connaît la colonie à partir des années 1830 . De ce point de vue, la lutte contre les sages-femmes et les charlatans nous semblent relever beaucoup plus de la légitimation idéologique de l'incorporation que de sa détermination historique. 
hôpitaux, de la place faite à la compétence et à l'expertise médicale dans l'administration hospitalière, de la mobilisation associative des médecins et finalement des organes de presse professionnels ${ }^{21}$.

L'une des caractéristiques importantes de cette phase est la prise de conscience brutale de l'urgence d'implanter des bureaux de santé municipaux et de voir à l'application des mesures de prévention en cas d'épidémies ${ }^{22}$. La passation de lois pour relancer la pratique de la vaccination obligatoire en $1853,1859,1861$ et 1866, compte elle aussi parmi les mesures qui tendent à faire consensus chez les médecins et les membres de l'Assemblée. On doit dire cependant que la médiocrité des fonds publics, la pauvreté assez généralisée de la population, la prédominance de l'économie de subsistance sur l'économie d'échange et la résistance à la taxation rendront particulièrement difficiles entre 1844 et 1872 la promotion de politiques sanitaires autres que circonstancielles. En fait, cette phase sera surtout celle où on tentera au moyen de la création des asiles d'aliénés, des hôpitaux de maternité, des hospices et des orphelinats privés d'aller au plus pressé des urgences sociales occasionnées par les vagues successives d'immigration. Ce n'est à vrai dire que vers 1865 que les écoles de médecine, les sociétés médicales et les périodiques montreront un relèvement de leurs effectifs et de leurs standards et que le corps médical se sentira davantage apte à revendiquer une véritable réforme des politiques sociales en matière de santé publique.

\section{c) La phase 1872-1894}

La phase 1872-1895 se démarque de la précédente par un brusque relèvement du palier de production - celui-ci passant de 20 à 90 textes

\footnotetext{
21 Ainsi, par exemple, en 1852, alors que McGill s'avère être la plus grosse école de médecine au Canada, les écoles de médecine québécoises récoltent pourtant moins d'étudiants, au total, qu'au Haut-Canada. Approximativement, les francophones ne comptent alors que pour $19 \%$ des étudiants inscrits en médecine dans les deux provinces et que pour $33 \%$ des étudiants inscrits dans les écoles du Bas-Canada. En 1860, ces chiffres passent respectivement à $23 \%$ et à $44 \%$. Au niveau associatif, les médecins anglophones font montre de plus d'initiative que les francophones. La Montreal Medico-Chirurgical Association est fondée en effet en 1843. Pratiquement unilingue, elle durera d'abord jusqu'en 1852 pour redémarrer, sur de meilleures assises, en 1865. La Société médicale de Québec quant à elle ne semble plus exister depuis longtemps que sur papier et ne renaîtra vraiment qu'en 1897. Quant à la Société médicale de Montréal, elle ne verra le jour qu'en 1871 et connaîtra de sérieuses difficultés de fonctionnement. Notons également, sur un autre plan, le refus des religieuses de l'Hôtel-Dieu de Montréal, encore au début des années 1850 , d'admettre dans leur hôpital, à des fins d'enseignement clinique, la présence des jeunes étudiants «laïcs» de l'École de médecine et de chirurgie. Cette pratique existe déjà pourtant couramment en milieu anglophone depuis la fondation du Montreal Medical Institution en 1823. Enfin, l'Hôpital Notre-Dame, fondé en 1880, sera le premier hôpital francophone d'importance à mettre les soins médicaux sous l'entière responsabilité des médecins, alors qu'en milieu anglophone ce sont les médecins qui ont pleine autorité sur les soins médicaux.

22 Notons en effet la création durant cette période de bureaux ou de commissions de santé à Montréal $(1847,1849,1852,1865)$ et à Québec $(1847)$ de même que la création d'un Bureau central de Santé (1854).
} 
par année de 1871 à 1872 - et par une croissance appréciable, bien que par à-coups, de l'information tout au long des années qui suivent. Au début des années 1890, la production d'information atteint en effet un sommet de 150 textes par année. Le brusque grossissement de volume qui inaugure cette nouvelle phase s'explique du fait que trois nouveaux médias littéraires paraissent simultanément la même année, en $1872^{23}$. Autre fait important, c'est qu'à compter de 1872 les périodiques tendent vers une plus grande stabilité et que la qualité de leur présentation (et très probablement leur tirage) s'améliore considérablement. Cela peut s'expliquer en bonne partie par l'augmentation très sensible du nombre des inscrits en médecine (et donc de praticiens consommateurs et producteurs d'informations) à partir de $1860^{24}$ et par une mise à profit plus systématique des périodiques et des ouvrages médicaux étrangers, les médecins anglophones de Montréal allant même jusqu'à former un Journal $\mathrm{Club}^{25}$ pour en faire plus systématiquement l'acquisition.

On peut compter aussi comme autres facteurs favorables à l'expansion du journalisme médical, la pénétration du marché médical anglophone du sud de l'Ontario qui s'industrialise ${ }^{26}$ et certains développements institutionnels majeurs qui viennent renforcer l'infrastructure de la profession: mentionnons la fondation de l'Association médicale canadienne (1867) qui tiendra des congrès annuels, la fondation de la faculté de médecine de Bishops (1871) dont l'organe officiel sera le Canada Medical Record et la seconde vague du mouvement hospitalier ${ }^{27}$ qui permettra l'exploration et l'expertise cliniques sur une plus vaste échelle. L'amélioration du curriculum d'enseignement, porté obligatoirement à quatre ans pour toutes les universités en vertu de la loi de $1876^{28}$, favorisera considérablement la qualification professionnelle des médecins et, par ricochet, la qualité du journalisme médical. Les voyages d'étude à l'étranger, qui deviennent pratique courante, y contribueront

\footnotetext{
23 C'est-à-dire L'Union médicale du Canada, le Canada Medical Record et le Canada Medical and Surgical Journal.

24 Entre 1860 et 1880 , le nombre de diplômés passe en effet de 30 à 90 par année. Il faut retenir toutefois qu'un bon pourcentage de ces diplômés iront pratiquer en dehors de la province de Québec. Voir George Weisz, op. cit., 133. Selon C. Roy, la faculté de médecine de l'Université Laval qui ne compte que 16 étudiants en 1857, en compte 51 en 1867 . Ce nombre passe à 72 dès 1876. En 1890 , le nombre des inscrits atteindra pratiquement 100 pour s'y stabiliser. Voir C. Roy, L'Université Laval et les fêtes du cinquantenaire (Québec, Typ. Dussault \& Proulx, 1903), 80 et 105 .

${ }_{25}$ Voir W. B. Howell, F. J. Shepherd-Surgeon, 101.

26 Il ne faut pas oublier en effet que la faculté de l'Université McGill sera l'une des clefs de voûte dans la continentalisation de la médecine et que son influence se fera vite sentir par le biais de ses diplômés.

27 La première vague renvoie aux années 1830-1850 où sont établis la quarantaine de la Grosse-Île, l'hôpital de la Marine, le Montreal Lunatic Asylum, l'asile de Beauport et les hôpitaux de maternité. Ces hôpitaux qui s'adressent tous à une clientèle démunie, à un prolétariat en formation, ont leurs exactes répliques en Angleterre et en France, mais au moins 70 ans plus tôt. Entre 1860 et 1895 , avec la deuxième vague, une trentaine d'hôpitaux seront mis en place.

28 Statuts de la province de Québec (1876), «Acte pour amender et refondre les actes concernant la profession médicale et la chirurgie dans la province de Québec».
} 
eux aussi. À compter des années 1880 , on remarque d'ailleurs que les comités de rédaction des périodiques sont devenus de véritables rouages administratifs et qu'apparaissent des rubriques davantage spécialisées, souvent confiées aux mêmes praticiens, ce qui contribue largement à la fixation et à la standardisation de la terminologie médicale ${ }^{29}$.

Notons enfin que la phase 1872-1895 est celle où se précipitent les grandes épidémies urbaines de variole, de diphtérie, de typhoïde et de tuberculose. Après l'ère du typhus et du choléra, immédiatement attribuable à la médiocrité des conditions de voyage en mer, les problèmes plus étroitement associés aux débuts de l'industrialisation et à la concentration des populations urbaines s'accentuent avec le mouvement migratoire des années 1870-1890: problèmes d'adduction d'eau, de drainage, d'évacuation des déchets organiques et chimiques (abattoirs, tanneries, filatures, fosses d'aisance, déchets domestiques et industriels), de mise en marché mal contrôlée des denrées périssables, de sous-alimentation, de logements insalubres et mal chauffés, de promiscuité et de mendicité, les «canicules» contribuant elles aussi à favoriser la propagation des infections (gastro-entérites) et la hausse du taux de mortalité. Il n'est pas étonnant que le périodique devienne alors un prolongement organique de l'institution médicale: les hôpitaux, qui se multiplient à un rythme rapide jusque dans les municipalités périphériques $^{30}$, requièrent et permettent en effet la diffusion d'une information médicale à caractère pratique, davantage axée sur l'étude des cas concrets et le suivi clinique que sur la maladie entendue comme entité nosologique formalisée. Les accidents de travail, plus nombreux en usines et sur les chantiers urbains et bien d'autres pathologies reliées au surmenage, aux mauvaises conditions de travail, au chômage, à la sous-alimentation et au stress psychologique viennent s'ajouter aux maladies contagieuses, devenues plus difficiles à contrôler particulièrement là où les mesures d'hygiène ne sont pas appliquées rigoureusement. Par la force des choses, l'hôpital, qui tendra progressivement à se subdiviser en départements spécialisés, devient le laboratoire par excellence de la pratique médicale. Au Montreal General Hospital, par exemple, le nombre de patients admis en clinique interne et externe passera de 12601 à 153892 entre 1873 et 1923 . C'est dans le va-etvient entre l'enseignement universitaire et la clinique «moderne», au

\footnotetext{
29 Grosso modo, on peut estimer que le mouvement de spécialisation commence à se dessiner au Québec autour des années 1875. La chaire d'ophtalmologie et d'otologie de l'Université McGill, par exemple, voit le jour en 1873. L'ophtalmologie, encore fusionnée avec l'otologie et la laryngologie, est, comme on le sait, la première spécialisation qui émerge après les trois champs traditionnels de la pratique médicale: la médecine, la chirurgie et l'obstétrique (doublée de la gynécologie). Suivront de près la pédiatrie et la dermatologie.

30 Ainsi, par exemple, l'Hôpital de Chicoutimi en 1884, l'Hôtel-Dieu d'Arthabaska en 1885, l'Hôtel-Dieu de Trois-Rivières en 1886, l'Hôpital de Sherbrooke en 1888 et l'Hôpital SaintJoseph de Rivière-du-Loup en 1889.
} 
sens où le capital des observations et des pratiques d'intervention est sans cesse consigné, revu et corrigé, que se forgeront désormais les grands noms de l'histoire médicale québécoise. Grossissement significatif de l'appareil hospitalier urbain, articulation systématique de l'enseignement académique sur la clinique hospitalière et essor du journalisme médical constitueront alors les trois facettes fonctionnelles d'une seule et même réalité. La phase 1872-1895 sera également celle où naîtront, pour éviter la trop grande surcharge des hôpitaux, les associations, les périodiques et les organismes d'hygiène, notamment la Société d'hygiène (1884) et le Conseil d'hygiène (1887) de la province de Québec. La multiplication des bureaux de santé locaux amènera elle aussi une modification importante dans le statut social du médecin: celle d'être un médiateur entre les classes pauvres et les classes riches, entre les marginalités et l'administration politique.

\section{d) La phase 1895-1900}

La phase 1895-1900, qui s'amorce avec la rénovation du Montreal General Hospital (1893-1896) et avec l'ouverture du Royal Victoria Hospital (1894), se caractérise par le passage, assez brusque lui aussi, à un quatrième palier de production. Dès la fin du XIXe siècle en effet, le nombre de textes produits annuellement sur les maladies infectieuses franchit la barre des 200. Ce nombre est alors de 10 fois supérieur à ce qu'il était durant la phase 1844-1871 et de deux fois supérieur à la production moyenne de la phase 1872-1894. Cette hausse est attribuable au fait que la presse médicale francophone connaît alors un regain de vigueur considérable avec la parution successive de $\mathrm{LaCli}$ nique (1894-1900), de La Revue médicale (1897-1904) et du Bulletin médical de Québec (1899-1936). Cette phase de production doit être mise en rapport avec la montée en flèche de l'information portant sur la tuberculose qui devient, à l'aube du XXe siècle, la préoccupation fondamentale des praticiens locaux et étrangers. Un autre élément important dans la croissance rapide de la production d'information médicale sur les infections en fin de siècle est sans contredit le triomphe généralisé dans l'hémisphère nord-occidentale des vues de Pasteur et de Koch qui révolutionnent littéralement le champ de la pathologie. L'isolement du bacille de la tuberculose par Koch (1882), la découverte par Klebs (1875) et la culture par Loeffler (1884) du bacille diphtérique, l'élaboration de la sérothérapie anti-tétanique par von Behring et Kitasato (1889) et les tentatives faites pour généraliser cette méthode à d'autres types d'infections, dont la diphtérie, la découverte par Eberth du bacille de la fièvre typhoïde (1880) et celle du vaccin antityphoïdique par Wright (1890), la mise au point du sérodiagnostic par Widal (1896), tout cela relance apparemment d'un seul coup l'intérêt médical des médecins québécois pour la pathologie des infections. Nul doute que les médecins locaux ont vu dans ce renouvellement de l'étiologie, 
du diagnostic et du traitement des maladies infectieuses une légitimation nouvelle pour la science médicale et un fondement infiniment plus solide aux principes de l'hygiène. Dès 1880 apparait en tout cas dans les périodiques un véritable engouement pour les nouvelles médications antiseptiques servies aussi bien sous forme d'onguents topiques que d'injections anales et sous-cutanées, de badigeonnage ou d'inhalation de vapeurs. Ce n'est cependant qu'à partir de 1894 que les laboratoires de pathologie clinique et de bactériologie deviennent partie prenante du diagnostic médical, encore que ce ne soit apparemment qu'à une échelle très modeste. Si on ajoute à cela le fait que le taux de mortalité opératoire et post-opératoire diminue sensiblement à compter des années 1890 en raison des progrès de l'anesthésie générale et en raison d'une amélioration sensible dans la formation pratique des chirurgiens ${ }^{31}$, que la radiologie se met au service de la médecine (1896) et que l'urologie (avec ses cystoscopies et ses colorants radio-opaques) et la cardiologie (avec ses électrocardiogrammes) tendent à devenir de véritables spécialités médicales entre 1905 et 1915 , on comprend que cette phase est, à proprement parler, celle où la médecine québécoise entre elle aussi, bien que sur le bout des pieds, dans l'ère de la médecine techno-scientifique.

\section{L'implication des médecins québécois dans la production d'informations sur les infections}

Le graphique 2 fait état du nombre de textes signés par les médecins québécois par rapport au nombre total des textes recensés. La signature signifie ici qu'un auteur prend à son compte les propositions qu'il produit et qu'il se trouve ainsi impliqué personnellement dans la production de l'information médicale. Il ne se contente pas de la répéter ou de la faire circuler. Il la synthétise et la communique sur la base d'une démarche intellectuelle qui lui est propre. Même si les idées avancées ne sont pas forcément originales, le signataire, qui se rend «responsable» de l'information, devient par le fait même un interlocu-

31 On peut présumer que la multiplication des grands hôpitaux, à compter de 1875 , dut permettre des interventions beaucoup plus nombreuses, une collaboration plus soutenue entre chirurgiens, une meilleure connaissance des processus physiologiques in vivo et une prise en compte plus systématique des réactions post-opératoires. Le recours à l'anesthésie générale est attesté à compter de 1848 mais de nombreux accidents sont attribuables à l'utilisation du chloroforme bien après cette date. En 1887, le docteur H. S. Birkett introduit l'inhalateur de Clover au Montreal General Hospital et ce n'est qu'en 1892 qu'un anesthésiologiste résident, le docteur Carmichael, y fait figure de spécialiste à plein temps. L'application du listerisme après $1877 \mathrm{au}$ Montreal General Hospital, contribue largement elle aussi à révolutionner la chirurgie qui, jusque dans les années 1880, fut plutôt considérée comme un art mineur. F. Shepherd mentionne qu'au cours des années 1880 , certaines interventions mobilisent jusqu'à sept chirurgiens. Les chirurgies plus risquées (trépanations, néphrectomies, thyroïdotomies, hystérectomies, laparotomies intestinales dans le cas de péritonites tuberculeuses ou typhoïdiques, etc...) se multiplient à compter des années 1890 . On estime qu'aux États-Unis, dans le cas de la chirurgie intra-abdominale, le taux de décès attribuable aux infections post-opératoires passe de 30\% à 6\% entre 1890 et 1915. 
teur possible pour l'ensemble de la profession. Sur une large échelle, la «signature» peut être indicative du degré d'implication explicite des membres d'une communauté scientifique donnée dans ses propres organes de presse.

On notera, de ce point de vue, que le degré d'implication des médecins québécois dans la production de l'information médicale sur les maladies infectieuses demeure très limité tout au long de la phase 1845-1870, les contributions annuelles signées par les praticiens locaux variant généralement entre 0 et un maximum de 6 . À compter de 1872 , on assiste à une hausse légère des textes signés, les deux pics des années 1872 et 1876 (tout comme celui de l'année 1885) étant davantage attribuables au climat polémique qui prévaut alors sur la question de la vaccination (sous forme d'échange de lettres parfois assez piquantes) qu'à une brusque recrudescence de l'intérêt proprement «scientifique» pour les maladies infectieuses. Paradoxalement, on peut dire qu'entre 1872 et 1886, l'écart entre le nombre de textes signés et le nombre total de textes tend à s'accentuer par rapport à la phase 1844-1871. Ce décalage est dû en bonne partie à l'importation croissante d'informations médicales étrangères et aux nombreux résumés, commentaires ou «gloses» anonymes qu'occasionne cette importation. Il n'est pas étonnant que la production typiquement locale soit dès lors noyée dans la masse globale de l'information. Il est important de remarquer cependant qu'entre les années 1885 et 1897 le nombre de contributions signées augmente progressivement, puisqu'il passe de 20 à 50 par année et que

GRAPHIQUE 2

Évolution du nombre de textes québécois signés par rapport au nombre total de textes recensés (1826-1899)

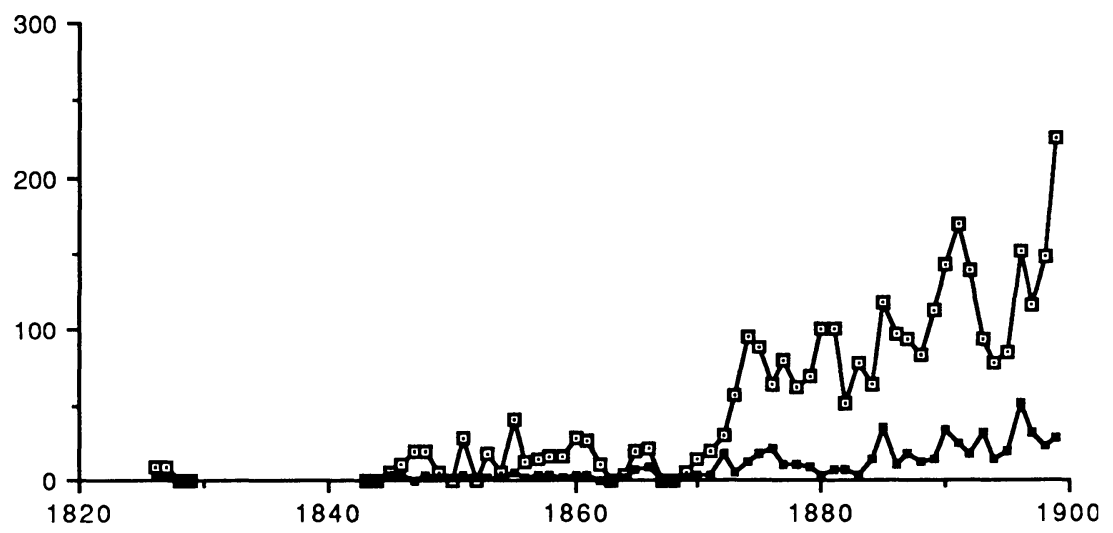


le parallélisme entre la courbe des textes signés et la courbe du total des textes mis en circulation tend à devenir constant. Cela peut suggérer que la médecine québécoise accède à un degré de maturité plus grand. En fin de siècle toutefois, l'importation massive d'informations sur les maladies vénériennes, la tuberculose et les fièvres typhoïdes, particulièrement par les nouveaux périodiques médicaux francophones en émergence, vient à nouveau modifier ce rapport. Comme si, avant de prendre son envol, la médecine francophone cherchait, à son tour, à se doter d'un sol ferme et d'une mémoire médicale. C'est, pourrait-on dire, l'époque de la francophilie médicale. Dans le sillage des médecins formés à l'Institut Pasteur et des sociétés médicales régionales qui se multiplient entre 1885 et 1900 , est d'ailleurs fondée en 1900 l'Association des médecins de langue française de l'Amérique du Nord. Cette association fait suite à la recréation de la Société médicale de Québec (1897) et à la renaissance de la Société médicale de Montréal (1900).

Le graphique 3 illustre le pourcentage de textes anglophones signés par rapport au nombre total de textes québécois signés. On en déduit que de 1845 à 1872, hormis quelques années d'exception, la production médicale anglophone l'emporte haut la main sur la production francophone. Entre 1872 et 1899 , plusieurs revirements momentanés s'opèrent en faveur des médecins francophones, notamment lors de l'inauguration de nouveaux périodiques et lors de l'épidémie de 1885 qui soulève un débat politique houleux sur la vaccination obligatoire. De façon générale cependant, le pourcentage des textes signés par les

GRAPHIQUE 3

Évolution en pourcentage des textes anglophones signés par rapport au total des textes québécois signés (1826-1899)

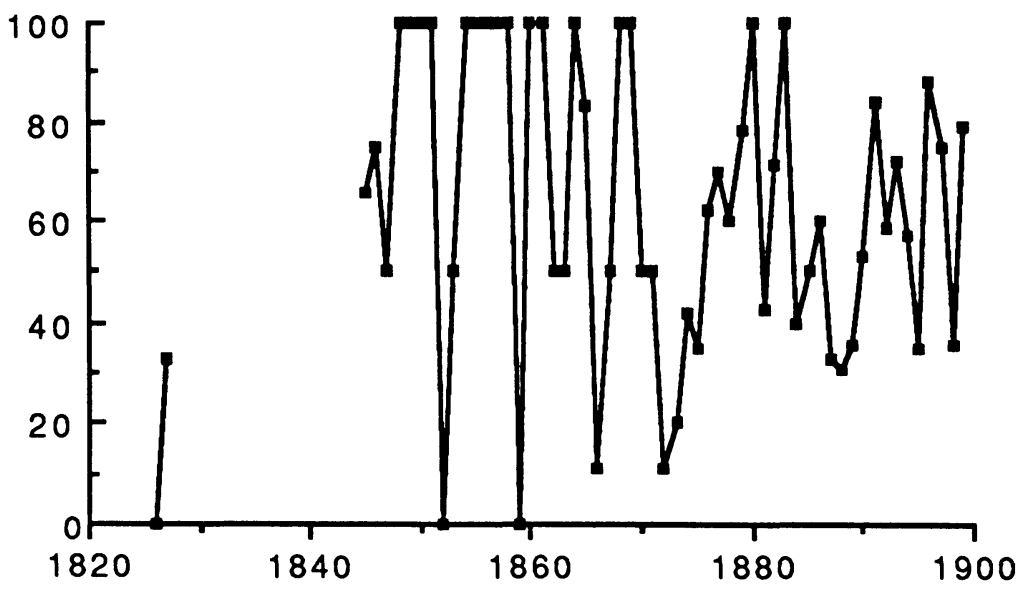


médecins anglophones ne tarde pas à redevenir prédominant. On constate en particulier qu'il atteint une autre apogée en 1892 et en 1896 . On peut dire cependant que, dans les cinq dernières années du siècle, le nombre de textes francophones signés tend progressivement à rejoindre celui des textes anglophones signés.

Si on comptabilise le nombre de textes francophones (signés et non signés) par rapport au nombre total de textes québécois (graphique 4), on constate de façon encore plus évidente l'absence à peu près complète d'information francophone entre 1844 et 1865 , exception faite de l'année 1847 qui voit naître la très éphémère Lancette canadienne. Cela se comprend lorsqu'on remarque la quasi-exclusivité de la présence anglophone dans le champ de l'édition des périodiques, au moins jusqu'à la fondation de L'Union médicale du Canada (1872). Cela nous amène à conclure que les médecins francophones ne disposent pas durant cette période d'organes médiatiques «spécialisés» comparables en nombre et en qualité à ceux de leurs collègues anglophones. On peut même se risquer à dire que ce n'est que vers 1885 que les médecins rédacteurs francophones commencent à développer de façon soutenue des habitus d'écriture dans leur discipline. Autre fait important, c'est que dès le début de la phase d'implantation (1844), et jusqu'à la toute fin du siècle, les périodiques médicaux se trouvent exclusivement concentrés dans la région de Montréal. En fait, il faudra attendre soixante-douze ans après la mort du Journal de médecine de Québec en 1827 , pour que naisse, dans la capitale provinciale, un deuxième pério-

\section{GRAPHIQUE 4}

Évolution du nombre de textes francophones par rapport au total des textes québécois (1826-1899)

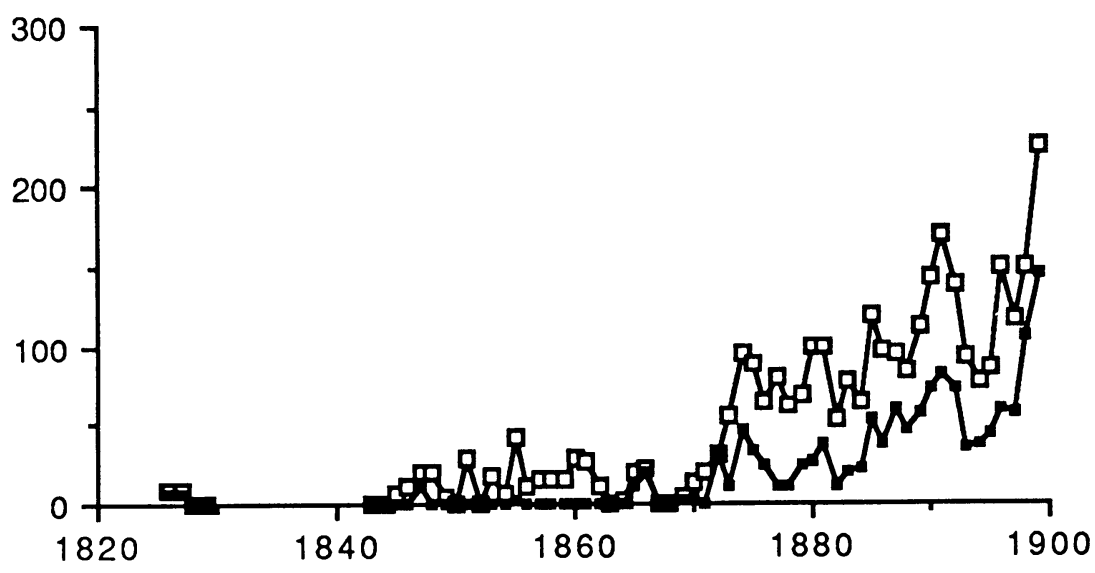


dique francophone. Partant de là, on pourrait s'interroger sur les inégalités de développement «régionales» entre la médecine francophone montréalaise (présumée plus «coopérante» avec le monde médical anglophone et international) et la médecine francophone de la capitale. Une étude comparative de la productivité littéraire des médecins de ces deux secteurs géographiques serait sans doute révélatrice de dynamiques fort distinctes tant dans le monde de l'institution médicale que dans celui des idées et de l'enseignement de la médecine. Notons enfin que c'est vers 1885 que s'amorce un certain équilibre entre le nombre global de textes médicaux francophones et anglophones.

\section{L'intérêt des médecins québécois pour les différentes pathologies infectieuses}

Les derniers graphiques que nous avons à proposer indiquent des variations et des écarts significatifs dans l'intérêt que portent les médecins aux différentes pathologies infectieuses tout au long de la seconde moitié du XIXe siècle. En d'autres termes, les maladies infectieuses, dont la plupart sont déjà connues grosso modo dès 1850 , ne présentent pas pour autant un intérêt égal aux yeux des médecins québécois pas plus que les différents champs de la pratique médicale n'ont une visibilité identique et constante tout au long du siècle. L'intérêt porté à telle ou telle maladie est largement conjoncturel et chaque maladie semble bien avoir son histoire propre. Comme le dit si bien Jacques Le Goff, «la maladie appartient à l'histoire d'abord parce qu'elle n'est qu'une idée, un découpage abstrait dans une réalité empirique complexe» et que «les maladies, en ce sens, sont mortelles» ${ }^{32}$. Pour nous, cela revient à dire que la production d'information médicale tient à l'intérêt pratique que l'on a de penser les phénomènes pathologiques et que cet intérêt, qui tient à des variables multiples, peut fluctuer et être redéfini d'une époque à l'autre. Pour des raisons aussi bien sociales que scientifiques, la médecine se développe de façon inégale, par déplacements et par bonds. Ainsi, par exemple, certaines maladies connaissent un plafonnement pauvre comme objets de savoir et semblent disparaître très tôt du champ de la nosographie. C'est, par exemple, le cas du typhus qui, dès les années 1850, ne semble plus présenter d'intérêt que marginal. D'autres pathologies, comme la syphilis et la tuberculose, connaissent au contraire une hausse de popularité croissante à compter des années 1870 tandis que la coqueluche connait une fortune relativement égale. Il faudrait évidemment chercher à savoir quels sont les facteurs qui peuvent rendre compte de ces fluctuations d'intérêt. Cela nous amènerait ultimement à développer une sorte de sociologie historique de la maladie où la relation entre la connaissance scientifique, l'histoire sociale

32 Voir Jacques Le Goff et J.-C. Sournia, Les maladies ont une histoire (Paris, L'histoire/ Seuil, 1985), 7. 
et l'institution médicale se trouverait pour ainsi dire soumise à l'autopsie. Selon le type de pathologies ou selon les champs de spécialisation pris en considération, nous aurions alors vraisemblablement autant de perspectives différentielles sur le développement historique de la médecine. Notre jugement sur l'évolution des intérêts de connaissance, des contextes d'évolution de la profession et du processus de médicalisation de la société s'en trouverait d'autant mieux éclairé.

$\mathrm{Si}$ on compare les trois premières figures du graphique 5 , on découvre trois profils nettement distincts dans la distribution des textes consacrées aux maladies infectieuses. Comme nous l'avons déjà dit, le typhus, qui est pourtant l'une des plus anciennes maladies de la colonie, accède à peine à l'état d'entité nosologique (notamment sous la plume des docteurs Badgley et G. Douglas en 1847-1848) qu'il disparaît presque aussitôt du champ de la littérature médicale québécoise. Ce n'est pourtant pas que la maladie n'avait pas de quoi inquiéter puisqu'en 1847 elle avait fait 8000 morts dans la région de Québec et 7000 dans la région de Montréal. On peut donc se demander à quoi peut bien tenir cette éclipse du typhus comme objet du discours médical.

Il peut y avoir plusieurs raisons à cela: d'abord, évidemment, l'absence d'épidémies et donc, possiblement, la rareté relative des cas cliniques observables après 1847-1848. Le typhus comptant parmi ces maladies qui se résorbent aussi vite qu'elles se répandent, on n'a guère le choix pour en reparler cliniquement que d'attendre qu'il se manifeste à nouveau. Il y a aussi le peu de contact que l'on entretient encore à ce moment avec les institutions médicales étrangères et le fait que la presse médicale locale, encore mal organisée, en est encore à ses premiers balbutiements. L'absence d'enracinement de la pratique médicale québécoise dans une écologie intellectuelle de longue durée, la difficulté par conséquent de s'élever au-dessus des circonstances immédiates, l'ampleur des priorités sociales et médicales propres à la colonie et le nombre restreint des médecins «rédacteurs» avant 1872 comptent sans doute aussi parmi les raisons de ce silence relatif qui entoure la pathologie du typhus.

Le cas du typhus doit donc être nettement distingué de celui du choléra (cf. graphique 5) qui, après les épidémies de 1832 et de 1834 , récidivera en 1849, 1851, 1852 et 1854 (de même qu'en 1866 en Europe, en 1873 et en 1891 aux États-Unis) maintenant la communauté médicale locale dans une continuelle expectative et à l'affût des signes «avantcoureurs» venant de l'étranger. Maladie nouvelle, encore inconnue de l'Occident avant 1832, et encore plus spectaculaire que le typhus dans ses effets, le choléra semble à ce point avoir marqué le siècle qu'on lui consacre encore, après la cinquième et dernière épidémie de $1854 \mathrm{au}$ Québec, un nombre appréciable de textes jusqu'en 1900. La maladie semble alors interpeller la profession médicale surtout lors des épidé- 


\section{GRAPHIQUE 5}

Évolution du nombre de textes sur le typhus, la variole, la tuberculose et le choléra (1826-1899)
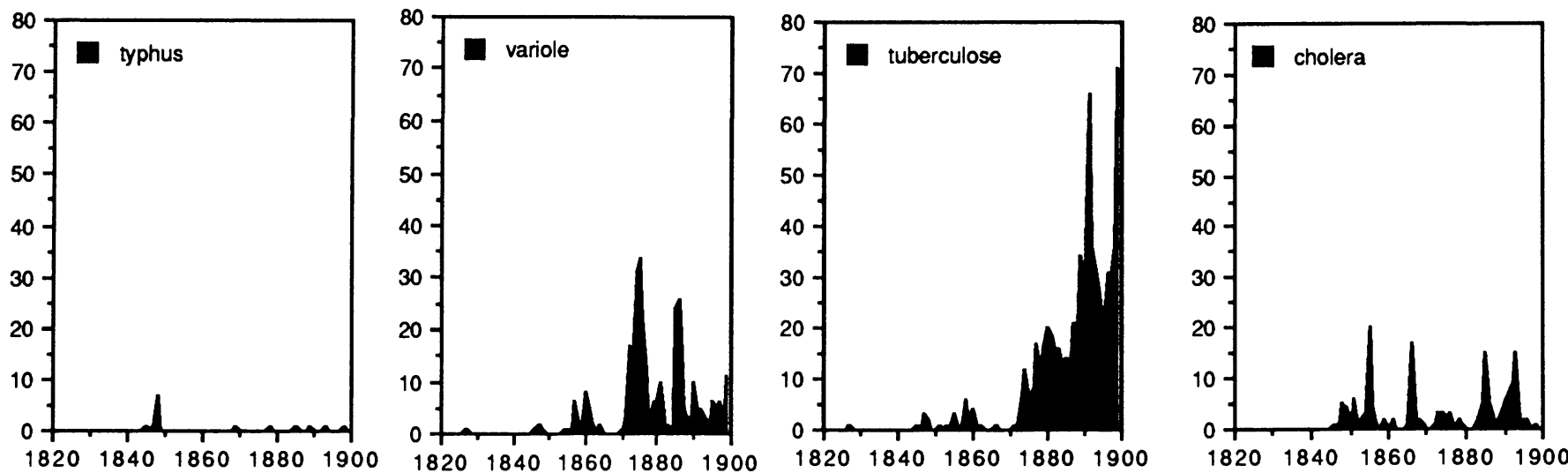
mies étrangères $(1866,1871$ et 1891$)$ et lors des grandes épidémies de variole locales (1872-1876 et 1885-1886), comme si une «catastrophe» en appelait (ou en rappelait...) forcément une autre. Le caractère généralement pandémique du choléra explique aussi en bonne partie qu'il ait été une occasion contraignante pour les autorités politiques et pour les médecins de la colonie de nouer des échanges d'informations continus avec le vieux continent. En témoignent la diffusion locale des recommandations du Central Board of Health de Londres en 1832, du Report of the London General Board of Health on Asiatic Cholera de 1849 de A. Savage, le Mémoire sur le choléra de J.-C. Taché en 1866, celui de W. F. Roome (1893) ainsi que la reproduction précoce de textes médicaux européens empruntés aux docteurs H. Sherrill (1835), M. Andral (1847), A. H. Stevens (1851), W. Fraser (1854) et John Snow (1855). Parmi les médecins locaux, ce sont les docteurs George Douglas (frère de l'aliéniste James Douglas), Chaperon, Marsden et W. Nelson qui seront les premiers à consacrer quelques articles à la pathologie du choléra. Notons enfin que la similarité de certains symptômes (diarrhée, déshydratation et état de choc septique) entre le choléra asiatique et le choléra infantum, gastro-entérite des canicules alors étroitement associée à la mortalité infantile, explique peut-être que le «fantasme» du choléra se soit imposé dans l'imaginaire de l'époque au point de «soutenir» l'intérêt des médecins bien après la dernière épidémie de $1854^{33}$. La rapidité de propagation du choléra, l'irrationnalité apparente de sa contagiosité, les coupures brutales qu'il introduit dans la sociabilité et les événements «mondains», son association à toutes sortes de croyances sur la moralité publique et les comportements individuels, comptent sans aucun doute aussi parmi les facteurs qui lui ont valu une notoriété et une visibilité beaucoup plus grande que d'autres maladies contagieuses, moins spectaculaires mais pourtant tout aussi mortifères, telle par exemple la pneumonie.

Le cas de la variole est tout autre. Maladie universelle et récurrente à tous les dix à douze ans, la variole est aussi la seule maladie contagieuse vis-à-vis de laquelle le corps médical du XIXe siècle dispose d'un «traitement» prophylactique efficace. La variole est donc, du point de vue de l'histoire de la profession, une maladie cible, une maladie «stratégique», puisque c'est autour de cette «fièvre éruptive» que se joue, métonymiquement en quelque sorte, l'image même de la profession. On s'explique donc les efforts soutenus qui ont été consacrés par les médecins du XIXe siècle à la promotion de politiques vaccinatoires et le fait que, déjà en 1827, certains médecins se plaignent, dans le Journal de médecine de Québec, de l'apathie de la population à cet égard. Mais la variole est aussi, de toutes les maladies, celle qui semble diviser le plus les médecins. Anciens et modernes, partisans de la théo-

33 C'est cette similarité qui amènera très vite l'américain Parrish à les distinguer. 
rie miasmatique et partisans de la découverte de Jenner, opposants et protagonistes de l'idée d'immunité (qui ne sera clairement discriminée en «active» et en «passive» qu'en 1892 avec Ehrlich), radicaux et modérés quant à la gravité des risques encourus par l'inoculation vaccinale, les médecins québécois du XIXe siècle finissent par s'opposer en deux clans assez bien circonscrits entre les années 1875 et 1885 . Mais le débat en réalité n'est pas que théorique. La difficulté d'établir un consensus sur une législation qui rendrait obligatoires la vaccination et la déclaration des varioleux est aussi à l'origine des tensions qui divisent les membres de la profession médicale. Autour de la variole se cristallise en fait une véritable querelle quant au droit de l'institution médicale d'intervenir dans la vie privée au nom de la santé publique. Le débat «scientifique» sur la nosologie et la prophylaxie de la variole se double ici, ouvertement ou en filigrane, d'un débat idéologique de fond sur la légitimité sociale de la prophylaxie «systématique» qui contrevient apparemment aux libertés individuelles et au précepte du secret professionnel.

On comprend dès lors l'intérêt soutenu porté à la variole dans les périodiques médicaux tout au long de la seconde moitié du XIXe siècle, mais plus particulièrement lors des épidémies de $1872-1876$ et de 18851886. Contemporain de l'affaire Riel, le débat ne va pas sans raviver de vieilles rancoeurs entre francophones et anglophones, les premiers accusant les seconds d'abuser des pouvoirs de la législature, les seconds reprochant aux premiers leur négligence inqualifiable. La mise et la remise sur pied d'un hôpital civique des varioleux montréalais soulève elles aussi de nombreuses discussions sur son financement, sur sa localisation et sur son caractère confessionnel. La rémunération des vaccinateurs publics et des fournisseurs de vaccins, tel le docteur Bessey, ne va pas sans émousser certaines sensibilités. Une fois adoptées les idées de Pasteur et mis sur pied des moyens plus adéquats de production et de conservation de la lymphe vaccinale, on constate toutefois (cf. graphique 5) que la variole perd passablement en importance et en intérêt et qu'elle passe rapidement au second plan par rapport à d'autres maladies, beaucoup plus «chroniques» et beaucoup plus critiques sur le plan épidémiologique. C'est notamment le cas de la fièvre typhö̈de (graphique 6), de la diphtérie (graphique 6) et de la tuberculose (graphique 5). Selon le rapport de la Commission royale de 1909-1910 sur la tuberculose, le Québec, qui de 1896 à 1906 ne compte plus que 240 personnes décédées de la variole, compte encore en contrepartie, pour la même période, 4450 décès pour la fièvre typhoïde, 12999 pour la diphtérie et 33190 pour la tuberculose. On comprend alors que ces trois maladies, pratiquement inexistantes dans la conscience médicale québécoise avant 1870, puissent monopoliser littéralement l'attention des médecins dès qu'apparaissent des conditions économiques tant soit peu favorables au développement de la clinique hospitalière. L'in- 
GRAPHIQUE 6

Évolution du nombre de textes sur la fièvre typhoïde, la diphtérie et la syphilis (1826-1899)
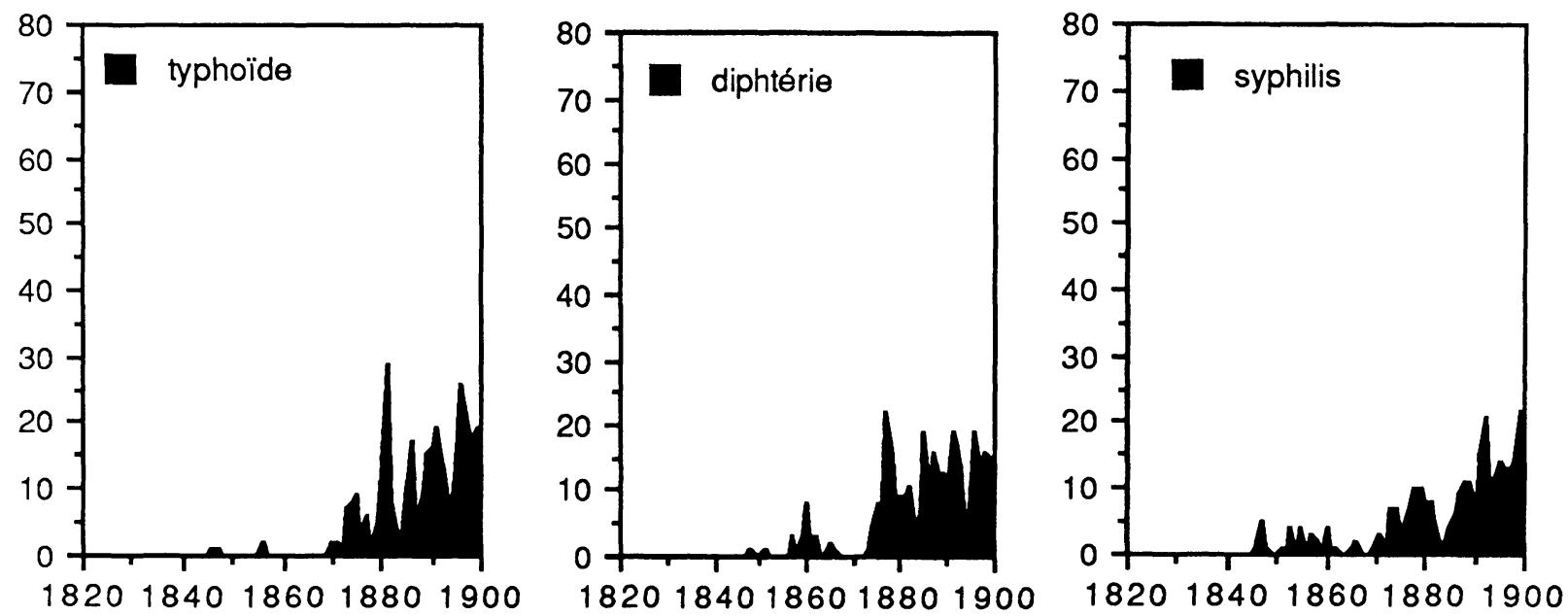
vestissement croissant, à compter de 1870 , dans la construction d'hôpitaux, dans la recherche clinique et thérapeutique sur les maladies sociales étroitement liées à la vie urbaine et dans l'hygiène de façon générale se comprend bien cependant si on admet qu'il ne s'agit plus exclusivement ici d'un simple combat humanitaire mené contre la fatalité naturelle (les microbes et la souffrance humaine), mais d'une véritable régulation systémique commandée par le développement même du capitalisme. Dans le contexte urbano-industriel de l'époque où, réquérant d'un côté une main-d'oeuvre vigoureuse et abondante on contribue de l'autre à générer des conditions de vie économiques et écologiques incompatibles avec un tel objectif, «l'entreprise» médicale devient, comme c'est le cas dans tous les pays en voie d'industrialisation, une véritable nécessité bio-sociale. Il faut aussi compter sur le fait que la maladie se calcule désormais en termes de coût social pour l'État, de risque pour les compagnies d'assurance, d'obstacle à la reproduction de la force de travail, de perte de salaire (et donc de profit) et de rendement en jours-travail pour les employeurs. En 1911, la commission sur la tuberculose rappellera que le grand total du coût de la tuberculose à Chicago pour la seule année 1908 s'élève à $23635190 \$$.

Le cas de la tuberculose (graphique 5) est d'autant plus intéressant que ce n'est apparemment qu'à compter du moment où la maladie est déjà socialement en voie de régression ${ }^{34}$ qu'on se met à réaliser de plus en plus son importance en Europe et aux États-Unis et à lui accorder une place sans précédent dans les périodiques médicaux de la fin du XIXe siècle. La Province de Québec présente toutefois cette particularité d'afficher encore un taux de mortalité relativement stable, celui-ci variant au bas mot autour de 192 tuberculeux par 100000 habitants selon les statistiques de l'époque. Dès le début des années 1870 , les médecins québécois consacrent à la tuberculose presque autant de textes qu'à la variole. À compter de l'année 1886, soit peu de temps après la découverte du bacille de la maladie par Koch, le nombre de ces textes passe très rapidement de 20 à 70 par année. Cette brusque profusion d'informations semble montrer que la maladie, «manifestement» contagieuse, suscite dès lors une vive inquiétude dans les milieux médicaux bien avant l'adoption des premières grandes mesures de prophylaxie sociale qui ne prendront effet que vers 1901 , peu de temps après que Flügge aura démontré que ce sont les poussières de salive rejetées par

\footnotetext{
34 Voir à ce propos R. H. Shryock, American Medical History, Historical Essays (Baltimore, The John Hopkins University Press, 1966), 25: «Tuberculosis mortality, strangely enough, began declining in England as early as 1850, and in «the States» after 1880». Voir aussi Y. Knibiehler, «La lutte antituberculeuse, instrument de la médicalisation des classes populaires (1870-1930)», La médicalisation en France du XVIIIe au début du XXe siècle, Annales de Bretagne et des pays de l'Ouest, 86,3 (1979): 333 : «Au point de vue biologique la lutte antituberculeuse n'était sans doute pas indispensable... En effet, l'acmé de la tuberculose se situe à la fin du XVIIIe siècle pour l'Angleterre, au cours du XIXe pour l'Europe continentale...»
} 
la toux qui sont responsables de la dissémination contagieuse des bacilles (1897). On imagine à quel point cette révélation dut renverser les préjugés «génétistes», alerter l'opinion publique et répandre la peur de voir les foyers contagieux s'étendre par le biais des rassemblements publics. La hantise du crachat n'est certainement pas exclusive à l'Europe... La lutte anti-tuberculeuse sera alors à l'origine de nombreuses initiatives médicales et para-médicales (commissions, ligues, dispensaires, hôpitaux et cliniques spécialisés, sanatoriums, services de radiographie scolaires et industriels, colonies de vacances, etc...) destinées à dépister les malades, à les soigner à domicile ou à les isoler de leur famille. Le Royal Edward Institute (1909), l'Institut Bruchési (1911), l'Hôpital Laval de Québec (1918) et la chaire de phthisiologie de la faculté de médecine de l'Université de Montréal (1913) sont quatre des premiers aboutissants de cet effort de mobilisation générale contre la maladie. Les sanatoriums, situés d'abord dans les Laurentides à compter de la toute fin du XIXe siècle, ne connaîtront par contre qu'une popularité médiocre au Québec et seront essentiellement destinés, ici comme ailleurs, à une clientèle aisée. Ce n'est finalement qu'à la fin des années 1920, qui s'amorceront avec l'implantation des services de diagnostic radiographique dans les dispensaires antituberculeux, que l'intervention médicale aboutira à des résultats probants: l'expérimentation du vaccin Guérin-Calmette par le docteur Beaudoin à compter de 1926, l'invention du pneumothorax artificiel par Bethune en 1931 et la pratique de la thoracoplastie par le docteur Archibald, chirurgien-en-chef de l'Hôpital Royal Victoria, comptent en effet parmi les premières formes de traitement efficace de la tuberculose.

L'intérêt tout à fait exceptionnel porté par les médecins du XIXe siècle à la tuberculose tient, comme nous l'avons vu, à des raisons économiques, mais aussi au fait que sa contagiosité, déjà postulée par Baumès en 1805 et prouvée par Villemin en 1865 , s'étend bien au delà des phases «limitées» et «passagères» que l'on connaît pour la plupart des maladies reconnues contagieuses à l'époque. À la fin du XIXe siècle, on calcule, sans doute pour cette raison, qu'on doit à la tuberculose près du tiers des décès occasionnés par l'ensemble des maladies, la gastro-entérite infantile étant la seule à lui damer le pion. L'intérêt exceptionnel porté à la tuberculose tient aussi au fait que ses symptômes, polymorphes, ne sont pas aisément reconnaissables et que la maladie - qui a une réputation honteuse et anti-eugénique - est aussi de celles que l'on n'ose dire quand on ne la masque tout simplement pas sous un autre nom: broncho-pneumonie, rhume négligé, catharre chronique, fluxion de poitrine... Tout cela rend évidemment ardu un diagnostic franc et précoce et représente une énigme de taille pour la profession médicale. Par ailleurs, cette maladie, aux symptômes multiples, requiert dans les cas déclarés une cure à moyen ou à long terme où peut être abondamment mise à profit la «technologie» de l'époque: le thermomètre, le sthétoscope pour l'auscultation et le microscope pour 
l'étude des crachats, des selles, des cultures de bacilles ou, plus largement, pour l'examen anatomo-pathologique. Pour la même raison, la tuberculose est aussi une maladie qui permet «optimalement» l'expérimentation thérapeutique: le traitement au chlorate de potassium, à l'eau froide, au calomel, au régime maigre, à l'huile de foie de morue, à l'arsenic et à la digitale, aux injections d'acide lactique, au gaiacol, au sulfonal, au thiocol et à la tuberculine ... comptent parmi les innombrables traitements dont il est fait mention dans les périodiques québécois entre 1860 et 1900 . En fait, on constate que la tuberculose, en raison de la pluralité de ses formes pathologiques, de ses localisations et de ses similitudes apparentes avec d'autres maladies (notamment la pleurésie et la pneumonie) est alors une véritable école de formation pour les praticiens québécois, que ce soit sur le plan du diagnostic différentiel et de la clinique, de l'anatomie pathologique ${ }^{35}$, de l'épidémiologie, de la microbiologie, de l'enseignement et de l'ouverture de la profession sur la médecine étrangère. Sans doute, le Québec du XIXe siècle ne compte pas de cliniciens aussi précoces que Bayle, Louis ou Baumès. Mais on ne peut oublier que déjà Osler s'y intéressait au tout début de sa carrière médicale, avant même sa nomination comme pathologiste au Montreal General Hospital en $1875^{36}$ et que la plupart des cliniciens québécois réputés du dernier tiers du siècle se sont intéressés étroitement au traitement de cette maladie: Adami, Blackader, Lemoine, Ross, Playter, Finley, Lafleur, McEachran, Johnston, Shepherd, Stewart, E.-P. Lachapelle et Laramée, pour n'en citer que quelques-uns, ont tous consacré en effet un ou plusieurs articles à la tuberculose. À plus d'un titre, et sans exclure pour autant d'autres maladies importantes comme la syphilis, qui mobilise aussi l'attention des médecins dans les vingt dernières années du siècle (graphique 6), on peut dire que la tuberculose doit être considérée comme la maladie qui a littéralement fait entrer la médecine québécoise dans l'ère de la clinique «moderne».

\section{CONCLUSION}

Ce bilan d'ordre quantitatif demeure très panoramique. Il établit néanmoins l'importance des infections comme centre d'intérêt croissant

\footnotetext{
35 Ainsi, par exemple, le laboratoire de pathologie de l'Hôpital Royal Victoria est inauguré en 1894. C'est apparemment à ce moment que l'anatomie pathologique connaît une véritable révolution puisque la même année $80 \%$ des décès au Royal Victoria, surtout des tuberculeux et des typhiques (typhoïde) sont autopsiés. Selon Lewis (voir Royal Victoria Hospital, 1887-1947 (Montreal, McGill University, 1969), 117), il s'agit là d'un record pour l'ensemble de l'Amérique du Nord. On n'ignore pas que les nombreux travaux d'Osler en anatomie pathologique publiés dans les Montreal General Hospital Reports avaient grandement ouvert la voie à cette curiosité pour l'étude des tissus et des organes malades.

36 Osler mentionne dans Aequanimitas que l'examen attentif des tissus tuberculeux occupait une place importante dans son apprentissage en anatomie pathologique dès 1871 , sous la direction du docteur R. P. Howard. La démonstration de la contagiosité de la tuberculose faite par Villemin le conduisit dès cette époque à lire Laennec, Graves et Stokes.
} 
tout au long du XIXe siècle et suggère que le développement de l'écriture médicale au Québec suit de très près les scansions qui marquent le passage de la société rurale à la société urbaine industrialisée. Si on prend les périodiques médicaux et les infections comme baromètre, notre bilan suggère aussi qu'après la période d' «ascension» des années 1800-1830 qui aboutit à la création de la Société médicale et du Journal de médecine de Québec, une cassure interrompt brusquement le développement des initiatives intellectuelles et que ce n'est qu'en 1845 que renaît le souci de penser la pratique, d'entraîner la profession sur la voie d'un savoir critique et professionnel. Mais ce sera cette fois sous l'égide presque exclusive de l'élite médicale anglophone de Montréal. Il faudra cependant attendre le début des années 1870 pour assister véritablement à l'envol de la presse médicale, et la grande épidémie de variole de 1885 pour voir les médecins francophones s'impliquer à leur tour de façon assidue dans des organes de presse qui leur soient propres. Notre bilan nous permet également de conclure, au moins provisoirement, que le nombre de médecins rédacteurs au XIXe siècle demeure, somme toute, très modeste par rapport à la masse globale des médecins pratiquant en terre québécoise. Formaliser les savoir-faire et les connaissances acquises en clinique, assimiler et diffuser ce qui se dit et se fait ailleurs, demeure encore apparemment le luxe d'une minorité de cliniciens qui pratiquent en milieu urbain et hospitalier, qui enseignent pour la plupart la médecine, et qui, jouissant de contacts plus étroits avec l'étranger, d'un cadre institutionnel plus stable et plus dynamique et de moyens financiers plus convaincants, peuvent initier le discours de la communauté médicale locale.

Ce bilan devrait évidemment nous inciter à chercher comment l'intérêt des médecins rédacteurs pour les infections a pu se traduire et se développer en termes de connaissances et de pratiques proprement médicales. Nous savons par exemple relativement peu de choses sur la nature exacte des différents types de traitements dispensés au XIXe siècle et sur les conceptions pathologiques souvent implicites sousjacentes à ces traitements. Leur nombre impressionnant nous incite à croire qu'ils relèvent d'une certaine gratuité empirique et qu'aucune théorie médicale ne les soutient. Mais c'est peut-être à tort. En réalité bien des hypothèses et bien des conceptions du corps, dans son rapport au milieu naturel et écologique, ont été formulées entre le début et la fin du XIXe siècle, depuis l'adhésion aux doctrines néo-hippocratiques jusqu'à l'adoption des tests permettant de porter des diagnostics bactériologiques précoces. Le temps de la médecine québécoise du XIXe siècle est un temps extrêmement contracté. Le passage de la médecine populaire et "philosophique» à la médecine professionnelle et scientifique s'y fait en l'espace de moins de 60 ans. On peut penser que les théories des miasmes, des animalcules et des germes, dans toutes leurs variantes plus ou moins spéculatives, ont connu une certaine vogue ici 
aussi. On ignore cependant à quels types de débats elles ont pu donner lieu. On ignore de même à quel rythme ont pu se développer les sciences chimique, biologique et pharmacologique au Bas-Canada et quel impact elles ont pu exercer sur la formation académique des médecins, sur leur pratique et sur leur approche de la maladie. Les influences étrangères et les canaux par lesquels ces influences ont pu pénétrer dans la colonie sont eux aussi mal connus parce que nous savons peu de choses finalement de l'itinéraire intellectuel et de la carrière professionnelle de nos praticiens. Peut-être y a-t-il des différences de pratiques et de discours importantes entre les médecins francophones et anglophones, particulièrement au niveau de l'enseignement de la médecine. Il y a peut-être aussi des formes d'échange et d'osmose que nous ignorons entre les deux communautés médicales qui, à première vue, semblent évoluer parallèlement, avec très peu de contacts, à compter de 1850 . Des recherches biographiques privilégiant ces dimensions aideraient surement à clarifier la question des sources et des influences tout comme elles nous permettraient de mieux comprendre les transformations du regard médical sur chaque type de pathologie infectieuse. Enfin, la part que prend l'imaginaire social et médical dans la formation des médecins et dans leur discours sur l'infection est, elle aussi, mal connue. Les valeurs morales, les croyances religieuses, les credo politiques et économiques, le surinvestissement de certaines conceptions et de certaines formes de réductionnisme médical (hygiénisme, chimisme, homéopathie, etc...) et de certaines médications (l'eau, l'air, l'éther, l'alcool, etc.), enfin tout ce qu'on peut ramasser sous le nom de «philosophie médicale» d'une époque, comptent pourtant parmi les ancrages culturels importants de la pratique médicale. Pour le meilleur et pour le pire, il n'y a ni médecins ni médecine qui transcendent l'univers symbolique et les stratégies socio-culturelles du milieu où l'on performe. En ce sens, si théorique soit-il, le discours des médecins sur la maladie doit toujours être pensé comme le discours d'intervenants sociaux en interaction. 